\title{
Leserumfrage zum Umgang mit knappen Mitteln in der Hämatologie und Onkologie
}

Dieses Themenheft fragt, welche Bedeutung der Kostendruck im Gesundheitswesen für die Hämatologie und Onkologie hat. Daher sollen hier unbedingt Sie zu Wort kommen. Welche Erfahrungen haben Sie gemacht? Erfolgt heute in der Versorgung hämatologisch-onkologischer Patienten* eine Rationierung? Kommt es zu einem Verzicht auf oder zu einer Verzögerung von nutzbringender Diagnostik oder Therapie? Wie entscheiden Sie in solchen Situationen und welche Kriterien sind für Sie bei der Entscheidung für eine teure Therapie wichtig?

Diese Umfrage wird vom AK Medizin und Ethik (http://www.dgho.de/informationen/dokumente-der-arbeitskreise/ak-medizin-und-ethik) durchgeführt und vom Vorstand der DGHO unterstützt. Der Fragebogen wurde auf der Grundlage von international durchgeführten Untersuchungen zu medizinethischen Aspekten der Verteilung von Mitteln im Gesundheitswesen entwickelt. Die Auswertung erfolgt selbstverständlich anonym.

In der DGHO arbeitet auch der Arbeitskreis $D R G$ und Gesundheitsökonomie zum Thema Kosten, Kostenerstattung und mögliche Rationierung. Eine Umfrage dieses Arbeitskreises zur aktuellen klinischen Praxis wird an alle DGHO-Mitglieder per E-Mail versandt. Wir möchten Sie herzlich bitten, die beiden Umfragen, die unterschiedliche inhaltliche Schwerpunkte haben, durch Ihre Teilnahme zu unterstützen. Die Studienergebnisse werden in Kooperation beider AKs ausgewertet.

Schließlich möchten wir Sie auch auf das «Critical Incidence Reporting System» (www.dgho.de/gesellschaft/cirs) hinweisen. Hiermit haben Sie die Möglichkeit, Fälle von Über- bzw. Unterversorgung in anonymisierter Form zu dokumentieren.

\section{Rücksendung des ausgefüllten Fragebogens}

Bitte trennen Sie den Fragebogen heraus (oder kopieren Sie ihn) und senden Sie ihn per FAX oder postalisch an

DGHO e.V. Hauptstadtbüro

AK Ethik

Alexanderplatz 1

10178 Berlin

Fax: 0302787 6089-18

Wenn Sie über das Ergebnis dieser Studie informiert werden möchten, dann senden Sie uns bitte eine E-Mail an jan.schildmann@ruhr-uni-bochum.de. Für Ihre Zeit und Bemühungen danken wir Ihnen sehr!

Jan Schildmann und Eva Winkler (für den AK Medizin und Ethik)

\footnotetext{
*In diesem Fragebogen wird aus Gründen der gebotenen Kürze und Lesbarkeit zur Bezeichnung gemischtgeschlechtlicher Gruppen die männliche Form verwendet. Gemeint sind stets beide Geschlechter, hier z. B. Patientinnen und Patienten.
} 
1. Die finanziellen Rahmenbedingungen (Anreize/Zwänge) führen im medizinischen Alltag tendenziell dazu, dass

\begin{tabular}{lcc} 
& Ja & Nein \\
\hline Nutzbringende Maßnahmen reduziert werden (Unterversorgung). & $\square$ \\
Maßnahmen mit fraglichem Zusatznutzen durchgeführt werden & $\square$ \\
(Überversorgung). & $\square$
\end{tabular}

2. Haben Sie im Rahmen Ihrer ärztlichen Tätigkeit einmal überlegt, auf eine möglicherweise nutzbringende diagnostische oder therapeutische Maßnahme ausschließlich aus Kostengründen zu verzichten?

Ja $\square \quad$ Nein $\square$ (Bitte gehen Sie direkt zu Frage 3)

Wenn ja, beschreiben Sie bitte ein konkretes Beispiel und die aus Ihrer Sicht ethischen Gründe für Ihre Überlegungen

3. Unsere Entscheidungen sind meist nicht von einem Faktor alleine bestimmt. Stellen Sie sich folgende

Situation vor: Für einen Patient käme aus medizinischer Sicht eine bestimmte Therapieoption in Frage.

Die Therapie ist zugelassen, allerdings betragen die Kosten ca. 3.000 Euro pro Therapiemonat. Wie stark würden Sie die folgenden Faktoren bei Ihrer Entscheidung für oder gegen die Durchführung dieser Therapie gewichten?

\begin{tabular}{|c|c|c|c|c|c|}
\hline & $\begin{array}{l}\text { stark } \\
\text { dafüir }\end{array}$ & $\begin{array}{l}\text { etwas } \\
\text { dafüir }\end{array}$ & $\begin{array}{l}\text { ohne } \\
\text { Einfluss }\end{array}$ & $\begin{array}{l}\text { etwas } \\
\text { dagegen }\end{array}$ & $\begin{array}{l}\text { stark } \\
\text { dagegen }\end{array}$ \\
\hline & ++ & + & 0 & - & -- \\
\hline $\begin{array}{l}\text { Lebensverlängerung von ca. } 2 \text { Monaten bei einem Patienten, } \\
\text { dessen Lebensqualität Sie als schlecht beurteilen. }\end{array}$ & $\square$ & $\square$ & $\square$ & $\square$ & $\square$ \\
\hline $\begin{array}{l}\text { Lebensverlängerung von ca. } 2 \text { Monaten bei einem Patienten, } \\
\text { dessen Lebensqualität Sie als gut beurteilen. }\end{array}$ & $\square$ & $\square$ & $\square$ & $\square$ & $\square$ \\
\hline $\begin{array}{l}\text { Deutliche Lebensqualitätsverbesserung bei einem Patienten, } \\
\text { dessen Lebenserwartung Sie mit ca. } 2 \text { Monaten veranschlagen. }\end{array}$ & $\square$ & $\square$ & $\square$ & $\square$ & $\square$ \\
\hline $\begin{array}{l}\text { Langzeitremissionen durch die Therapie in statistisch } 10 \% \\
\text { der Fälle. }\end{array}$ & $\square$ & $\square$ & $\square$ & $\square$ & $\square$ \\
\hline $\begin{array}{l}\text { Sie denken zwar, der Patient könnte profitieren, aber die } \\
\text { Datenlage ist nach Ihrer Einschätzung nicht ausreichend. }\end{array}$ & $\square$ & $\square$ & $\square$ & $\square$ & $\square$ \\
\hline Der Patient hatte bisher keine krankheitsspezifische Therapie. & $\square$ & $\square$ & $\square$ & $\square$ & $\square$ \\
\hline Die Krankheit ist auf Rauchen/Alkohol zurückzuführen. & $\square$ & $\square$ & $\square$ & $\square$ & $\square$ \\
\hline $\begin{array}{l}\text { Die Therapie wird in Leitlinien für diese Therapiesituation } \\
\text { empfohlen. }\end{array}$ & $\square$ & $\square$ & $\square$ & $\square$ & $\square$ \\
\hline Sie haben Sorgen wegen haftungsrechtlicher Gesichtspunkte. & $\square$ & $\square$ & $\square$ & $\square$ & $\square$ \\
\hline
\end{tabular}




\begin{tabular}{|lccccc} 
& $\begin{array}{l}\text { stark } \\
\text { dafür }\end{array}$ & $\begin{array}{l}\text { etwas } \\
\text { dafür }\end{array}$ & $\begin{array}{l}\text { ohne } \\
\text { Einfluss }\end{array}$ & $\begin{array}{l}\text { etwas } \\
\text { dagegen }\end{array}$ & $\begin{array}{l}\text { stark } \\
\text { dagegen }\end{array}$ \\
$\begin{array}{l}\text { Der Patient informiert sich gut über Therapiemöglichkeiten } \\
\text { (z.B. Internet). }\end{array}$ & $\square$ & $\square$ & $\square$ & $\square$ & $\square$ \\
\hline $\begin{array}{l}\text { Der Patient ist unter 40. } \\
\text { Der Patient ist über 80. }\end{array}$ & $\square$ & $\square$ & $\square$ & $\square$ & $\square$ \\
\hline Der Patient ist multimorbide/gebrechlich. & $\square$ & $\square$ & $\square$ & $\square$ & $\square$ \\
\hline Der Patient ist kognitiv eingeschränkt. & $\square$ & $\square$ & $\square$ & $\square$ & $\square$ \\
Der Patient hat eine wichtige Position in der Gesellschaft (,VIP“). & $\square$ & $\square$ & $\square$ & $\square$ & $\square$ \\
\hline Der Patient hat ein starken Therapiewunsch. & $\square$ & $\square$ & $\square$ & $\square$ & $\square$ \\
\hline Der Patient ist privat versichert. & $\square$ & $\square$ & $\square$ & $\square$ & $\square$ \\
\hline
\end{tabular}

Erläuterungen:

4. Stellen Sie sich vor, dass aufgrund der begrenzten Mittel im Gesundheitswesen nicht mehr alle Maßnahmen mit einem nachgewiesenen Nutzen von der Gesetzlichen Krankenversicherung übernommen werden können (Rationierung). Es stehen nun zwei Rationierungsstrategien zur Auswahl. Bitte entscheiden Sie sich für eine der beiden Möglichkeiten:
$\square \quad$ Medikamente mit geringer Effektivität (z.B. weniger als 3 Monate Lebenszeitgewinn) sollen unabhängig vom Preis grundsätzlich nicht erstattet werden.
$\square \quad$ Medikamente mit geringer Effektivität (z.B. weniger als 3 Monate Lebenszeitgewinn) sollen nur dann nicht erstattet werden, wenn sie teuer sind (z.B. $>3000 € /$ Monat). Wenn sie billig sind, sollen sie erstattet werden.

Erläuterung/Meinung:

Demografische Angaben (Die Auswertung erfolgt anonymisiert!)

1. Alter (in Jahren)

2. Geschlecht: Weiblich $\square \quad$ Männlich

3. Bundesland:

4. Tätigkeit in: Praxis $\square$ Klinik $\square$ andere:

5. Bei Tätigkeit in einer Klinik:
Assistenzarzt
$\square$
Oberarzt
Universitätsklinik
Trägerschaft:
Maximalversorgung
Gemeinnützig
kleines/mittl. Krankenhaus
Staatlich
Privat
Belegarzt
$\square$ 